\title{
The alignment of nematic liquid crystal by the Ti layer processed by nonlinear laser lithography
}

\author{
Ihor Pavlovi,b, Andrey Rybak ${ }^{\mathrm{b}}$, Andrii Dobrovolskiyc, Viktor Kadan ${ }^{\mathrm{b}}$, Ivan Blonskiy ${ }^{\mathrm{b}}$, Fatih Ö. Ildaya,d,e, \\ Zoya Kazantseva ${ }^{f}$ and Igor Gvozdovskyy ${ }^{g}$
}

aDepartment of Physics, Bilkent University, Ankara, Turkey; 'bepartment of Photon Processes, Institute of Physics of the National Academy of Sciences of Ukraine, Kyiv, Ukraine; 'Department of Gas Electronics, Institute of Physics of the National Academy of Sciences of Ukraine, Kyiv, Ukraine; ${ }^{d}$ Department of Electrical and Electronics Engineering, Bilkent University, Ankara, Turkey; eUNAM - National Nanotechnology Research Center and Institute of Materials Science and Nanotechnology, Bilkent University, Ankara, Turkey; fElectrical and Galvanomagnetic Properties of Semiconductors, V.E. Lashkaryov Institute of Semiconductor Physics of the National Academy of Sciences of Ukraine, Kyiv, Ukraine; 'Department of Optical Quantum Electronics, Institute of Physics of the National Academy of Sciences of Ukraine, Kyiv, Ukraine

\begin{abstract}
It is well known that the alignment of liquid crystals (LCs) can be realised by rubbing or photoalignment technologies. Recently, nonlinear laser lithography (NLL) was introduced as a fast, relatively low-cost method for large area nano-grating fabrication based on laser-induced periodic surface structuring. In this letter for the first time, the usage of the NLL as a perspective method of the alignment of nematics was presented. By NLL, nanogrooves with about $0.92 \mu \mathrm{m}$ period were formed on Ti layer. The nanostructured Ti layer (NSTL) was coated with oxidianilinepolyimide film with annealing of the polymer followed without any further processing. Aligning properties of NSTLs were examined with combined twist LC cell. The dependencies of the twist angle of LC cells and azimuthal anchoring energy (AE) of layers on scanning speed and power of laser beam during processing of the Ti layer were the focus of our studies as well. The maximum azimuthal $A E$, obtained for pure NSTL, is comparable with photoalignment technology. It was found that the deposition of polyimide film on NSTL leads to the gain effect of the azimuthal AE. Also, atomic force microscopy (AFM) study of aligning surfaces was carried out.
\end{abstract}
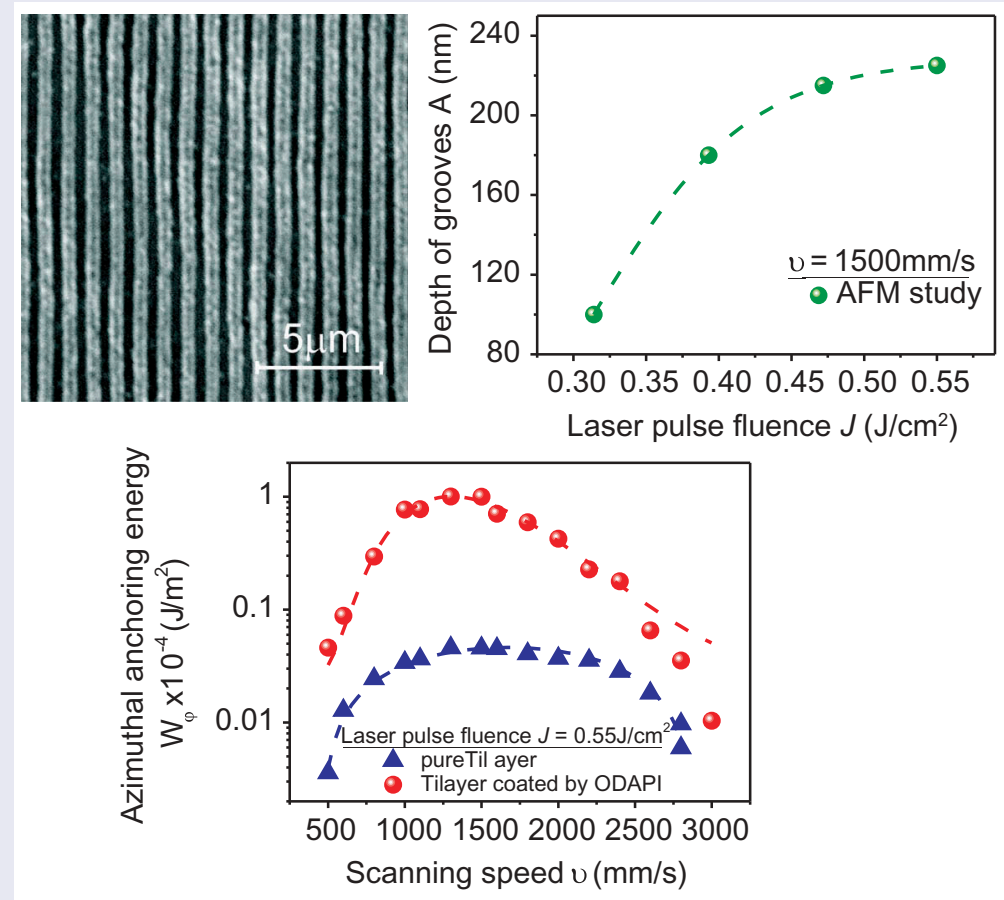

CONTACT Igor Gvozdovskyy igvozd@gmail.com

๑ 2018 Informa UK Limited, trading as Taylor \& Francis Group

\section{ARTICLE HISTORY}

Received 23 November 2017 Accepted 14 January 2018

\section{KEYWORDS}

Nonlinear laser lithography; nanostructured titanium layers; polyimide; aligning layers; nematic liquid crystals; azimuthal anchoring energy 
This letter is dedicated to the memory of the great scientist, Prof. Yu. A. Reznikov, who was among the first to discover the photoalignment effect of liquid crystals.

\section{Introduction}

The alignment of liquid crystals (LCs) is important and a key condition for their application as a liquid with anisotropic properties. For this purpose, both different aligning materials and various methods of their processing can be used, as demonstrated, for instance, in the book [1] and many reviews [2-5]. However, in present two main methods can be emphasised, which are used for the creation of the aligning layers and were well studied for further application in display technology, etc. The first method, used extensively for different applications in industry, is the rubbing technique with various materials [1,6-9] of different surfaces $[1,10]$. Despite the fact that the rubbing technology is widely used in liquid crystal display (LCD) technology, however this technique has some shortcomings, among which are accumulation of both the static charges and dust particles [4]. The other method of the homogeneous aligning of LCs is a recently discovered photoalignment effect [11-15]. It is a real alternative method to the rubbing technique, because the usage of photosensitive materials, deposited on a substrate or dissolved in the bulk of LCs, leads to the change of the orientational order of photoproducts under polarised light irradiation. In this case the mechanical contact with the surface of a substrate, with all the shortcomings that this entails [4], is absent. However, photoaligning materials often possess photodegradation, and in order to create a relatively large area of aligning surfaces there is a need for a long-term exposure and preparation of masks. As it was noted in Ref. [4], the photoalignment technology gives an effective control of main anchoring parameters, namely: easy orientation axis, pretilt angle and anchoring energy (AE). It is noted that depending on chemical properties of materials $[2,4,16], \mathrm{AE}$ values of the photoaligning surfaces can be within a wide range $10^{-8}-10^{-4} \mathrm{~J} / \mathrm{m}^{2}$.

In the case of both methods, the formation of the aligning layers can be created by means of deposition of inorganic materials, by polymer-coating from different solutions, by using of Langmuir-Blodgett, spin-coating and dipping techniques, followed by polymerisation and their further processing with application of a rubbing roll or polarised light. In addition, it should be noted that the usage of plasma beam as a method of processing aligning layers for the homogeneous orientation of LC was recently studied $[17,18]$. After a certain method of the processing of the aligning layers, it was observed that the period of the grooves structure can change within a range 100-300 $\mathrm{nm}$, while the amplitude (depth) of relief can be within a range $80-150 \mathrm{~nm}$ $[6,7,19,20]$. The homogeneous alignment of the LC cell was observed due to, on the one hand, the appearance of the anisotropic properties of aligning layers and, on the other hand, long-range interaction of the LC molecules in a mesophase.

In addition, to obtain the LC alignment by a surface with a small period (about $235-250 \mathrm{~nm}$ ) of nanogrooves the e-beam lithography [21] and atomic force microscopy (AFM) nano-rubbing [22] were applied. However, the area of nanogrooves rubbed on the surface was very small [21], and both methods show a very low throughput. Moreover, the nano-imprint lithography [23] and photolithography [24] were also used to create nanogrooves on a polymer surface for aligning LCs, but both techniques are complicated due to the preparation of masks.

It should be also noted that recently, the fast and high-throughput method, consisting of the splitting of a polymer film with further propagation of the wave front to induce self-assembled micro and nanogrooves on a polymer surface (the so-called crack-induced grooving method), was proposed to align LCs [25].

The possibility of processing of the various materials by ultrafast laser pulses for different applications is described in detail [26]. In this letter, for the first time we have made aligning layers, using a simple, relatively low-cost and high-speed method of the creation of a large area of high anisotropic metal-oxide periodic nanostructures (the so-called ripples) with femtosecond laser [27]. Conditionally, it can be done in one or two stages. Contrary to the traditional methods [1-20] of the LC alignment, at the first stage the processing of titanium (Ti) layer, deposited on a glass substrate, which results in the periodic nanogrooves with certain parameters (for instance, depth, period and angle of direction of ripples), is realised. At the second stage, the creation of aligning surfaces owing to the coating of a polymer onto a layer with $\sim 0.92 \mu \mathrm{m}$ period of nanogrooves without additional processing of a polymer (such as rubbing or irradiation with polarised light), excepts the process of polymerisation. The calculation of $\mathrm{AE}$ of both the pure nanostructured Ti layer (NSTL) and similar layer with the polymercoated surface were carried out on the basis of the measurement of the twist angle in the combined twist LC cell $[28,29]$. The dependence of the calculated azimuthal $\mathrm{AE}$ on the scanning speed and laser pulse fluence (LPF) during periodic Ti surface structuring was studied. 


\section{Experiment}

To measure the twist angle and calculate AE herein the idea of combined twist LC cells, consisting of the tested and reference substrates was used [28-30].

The tested substrates are used of two types. The first type of the tested substrate was coated with $300 \mathrm{~nm} \mathrm{Ti}$ layer and further processed by the method of nonlinear laser lithography (NLL), using the laser scanning with femtosecond pulse [27] $(P=350 \mathrm{~mW}, f=1 \mathrm{MHz}$ and $I=350 \mathrm{~nJ})$. The direction of nanostructures was inclined at a fixed angle of $8^{\circ}$ to the one side of the substrate.

The second type of the tested substrate consists of the first type substrate additionally coated with $1 \%$ dimethylformamide (DMF) solution of oxidianilinepolyimide (ODAPI) (Kapton synthesised by I. Gerus, Ukraine) by means of dipping technique using equipment for Langmuir-Blodgett film preparation R\&K (Wiesbaden, Germany). The second type of tested substrate was dipping into appropriable solution and further vertical drawing up at constant speed about $5 \mathrm{~mm} / \mathrm{min}$ along direction of the NSTLs.

The reference substrate was coated with polyimide PI2555 (HD MicroSystems, USA) processed with the rubbing technique having strong $\mathrm{AE} \mathrm{W}=(4 \pm 1) \times 10^{-4} \mathrm{~J} / \mathrm{m}^{2}$ for the certain pressure and number of times of unidirectional rubbings [31,32]. The rubbing was carried out at $45^{\circ}$ to one side of the substrate. The thickness of a gap was set to $20-25 \mu \mathrm{m}$ by a Mylar spacer and measured by the interference method, using transmission spectra of empty LC cells. The LC cells were filled with the nematic LC E7 (obtained by Licrystal Merck, Germany) with twist elastic constant $\mathrm{K}_{22}=6.8 \mathrm{pN}$ [33], at the higher temperature $T=61^{\circ} \mathrm{C}$ than the temperature of the isotropic phase $\left(T_{\text {Iso }}=58^{\circ} \mathrm{C}\right)$ and slowly $\left(\sim 0.1^{\circ} \mathrm{C} / \mathrm{min}\right)$ cooled.

To measure the twist angle $\varphi$, the simple scheme described in [30] was used. For this the output of monochromatic linear-polarised light $(\lambda=632.8 \mathrm{~nm}$ and power about $1.5 \mathrm{~mW}$ ) from $\mathrm{He}-\mathrm{Ne}$ laser LGN207a (Lviv, Ukraine) and a silicon photodetector (PD) FD-18K (Kyiv, Ukraine) with the spectral range 470$1100 \mathrm{~nm}$ were chosen. PD was connected to the oscilloscope Hewlett Packard 54602B $150 \mathrm{MHz}$ (USA).

\section{Results and discussion}

Figure 1(a) presents the scanning electron microscope (SEM) image of the pure NSTL with a high degree of anisotropy. The dependence of the nanogrooves depth A on LPF J, showed in Figure 1(b), has analogy with the results in Ref. [20], where the influence of exposure
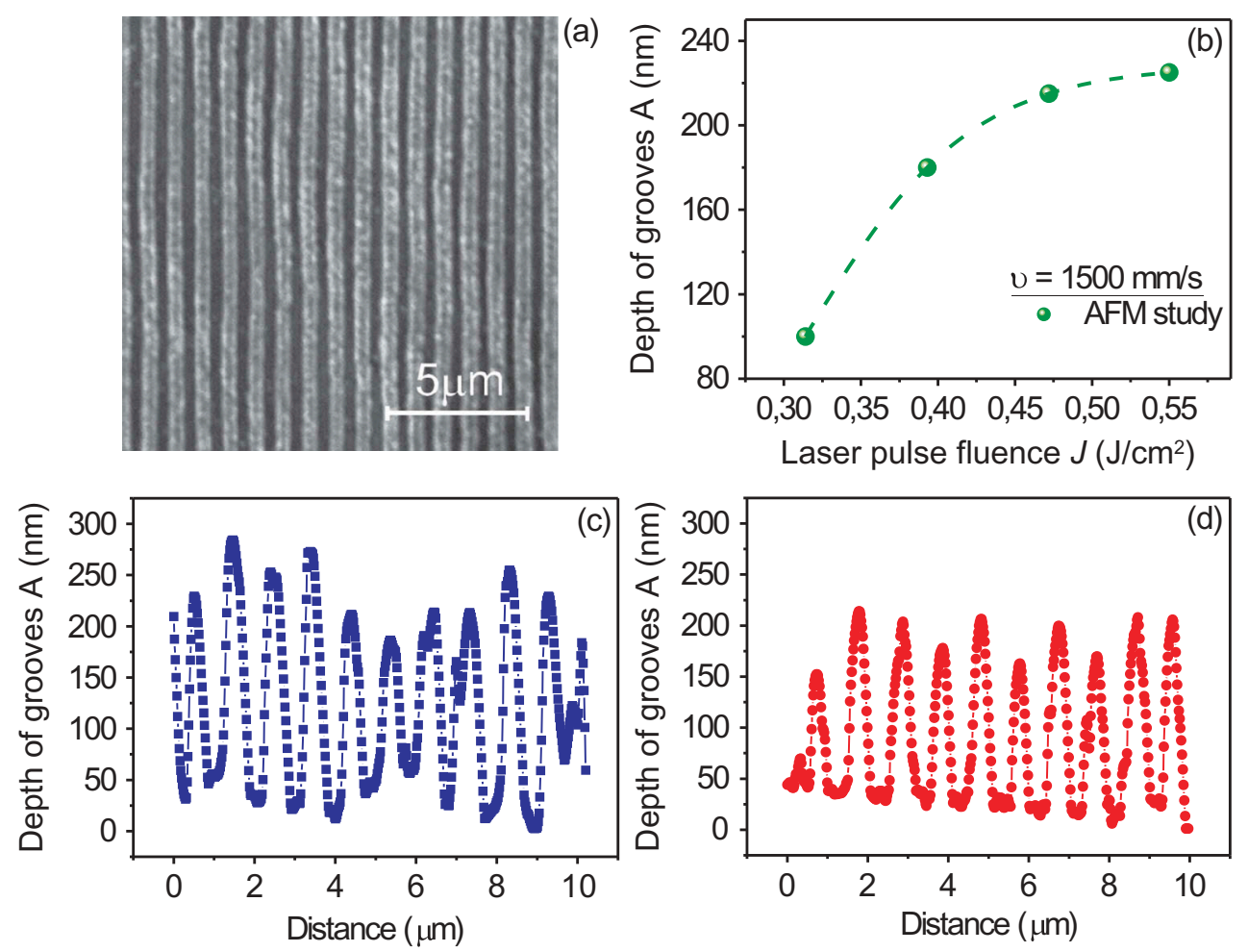

Figure 1. (Colour online) (a) SEM image of the pure NSTL after processing with the NLL method. (b) Dependence of the average depth A on the LPF $J$ of the pure NSTL after the NLL processing. The cross section of nanogrooves of the Ti layer: (c) pure, possessing the average period $\Lambda=0.92 \mu \mathrm{m}$ and average depth $A=225 \mathrm{~nm}$ and (d) coated with the ODAPI film, having the average period $\Lambda=0.92 \mu \mathrm{m}$ and average depth $A=175 \mathrm{~nm}$. In the NLL method, the LPF $J$ and scanning speed $U$ was $0.582 \mathrm{~J} / \mathrm{cm}^{2}$ and $1500 \mathrm{~mm} / \mathrm{s}$, respectively. 
on the value of the grooves depth during the photoaligning technique was studied. The cross section of nanogrooves of the pure NSTL obtained by AFM is shown in Figure 1(c). At constant parameters of the NLL $\left(J=0.55 \mathrm{~J} / \mathrm{cm}^{2}\right.$ and $\left.v=1500 \mathrm{~mm} / \mathrm{s}\right)$ by AFM study it is found that the certain periodic structure with an average period $\Lambda=0.92 \mu \mathrm{m}$ and average depth $A=225 \mathrm{~nm}$ is formed. In contrast to methods traditionally used for the alignment of LCs $[6,7,19,20]$, where the period of nanogrooves is within a range $100-300 \mathrm{~nm}$, it can be assumed that owing to anisotropy of NSTLs the homogeneous alignment of the nematic LC may be observed, however, AE of the layers will be lower.

To estimate the order of the AE value of pure NSTLs we use the Berreman's theory [6]. According to this theory, the AE depends on the depth A and period $\Lambda$ of nanogrooves, and can be written as $[6,19,20]$ :

$$
W_{B}=2 \pi^{3} \times A^{2} \times K_{22} / \Lambda^{3}
$$

Thus, by using both period and depth of nanogrooves measured by AFM, the value $W_{\mathrm{B}}$ of the pure NSTL reaches $\sim 2.7 \times 10^{-5} \mathrm{~J} / \mathrm{m}^{2}$. This value is of order of the azimuthal $\mathrm{AE}$ of azopolymers [2,16], photo-crosslinking materials [2,5,34-37] and polyvinylcinnamate [36,37]. According to the theory [6], to increase the $\mathrm{AE}$ value of the pure Ti layer, it is necessary either to decrease the period, or to increase the depth of the nanogrooves.

However, here to increase the $\mathrm{AE}$ value we propose not to change the main parameter, such as the wavelength of a laser, resulting in the change of the nanogrooves period, during the surface structuring with the NLL method. But we suggest additionally apply a coating of the ODAPI film onto the surface of the NSTL. As can be seen from Figure 1(d), the average period and average depth of nanogrooves was about $0.92 \mu \mathrm{m}$ and $175 \mathrm{~nm}$, respectively. According to Berreman's theory [6], the calculated $\mathrm{AE}$ value decreased to $1.7 \times 10^{-5} \mathrm{~J} / \mathrm{m}^{2}$, owing to the insignificant change of the average depth of nanogrooves after the coating of the polymer film (Figure 1(d)).

Let us consider the real AE value measured experimentally, using the method of the combined twist LC cell [28-30].

The preparation of the combined twist LC cells, consisting of the tested substrate, having NSTLs of both types, and reference substrate with a rubbed PI2555 layer, and measurement of the twist angle $\varphi$ of the sample allow us to calculate the $\mathrm{AE}$ value $\left(W_{\varphi}\right)$ of the aligning layer of the tested substrates. According to Refs. [28,29,37], the twist angle $\varphi$ is related to the azimuthal $\mathrm{AE}_{\varphi}$ as follows:

$$
W_{\varphi}=K_{22} \times \frac{2 \times \sin (\varphi)}{d \times \sin 2\left(\varphi_{0}-\varphi\right)}
$$

where $d$ is the thickness of the LC cell, $\varphi_{0}=36^{\circ}$ is the angle between the easy axes of the reference and tested substrates and $\varphi$ is the measured twist angle.

Owing to the NLL method [27], allowing to do changes in a wide range of the scanning speed $v=500-3000 \mathrm{~mm} / \mathrm{s}$ and LPF $J$ within a range $0.236-$ $0.59 \mathrm{~J} / \mathrm{cm}^{2}$, dependencies of $\varphi(v)$ and $\varphi(J)$ were studied. For both types of the tested substrates, dependencies $\varphi$ $(v)$ and $\varphi(J)$ are shown in Figure 2(a,b), respectively. As can be seen from Figure 2, the twist angles of the LC cell are reaching maximum values at a certain range of values of $v=1300-2000 \mathrm{~mm} / \mathrm{s}$ or $J=0.393-0.582 \mathrm{~J} / \mathrm{cm}^{2}$ for both types of the tested substrates.

The dependence of the calculated azimuthal AE of the aligning film on the scanning speed $v$ during

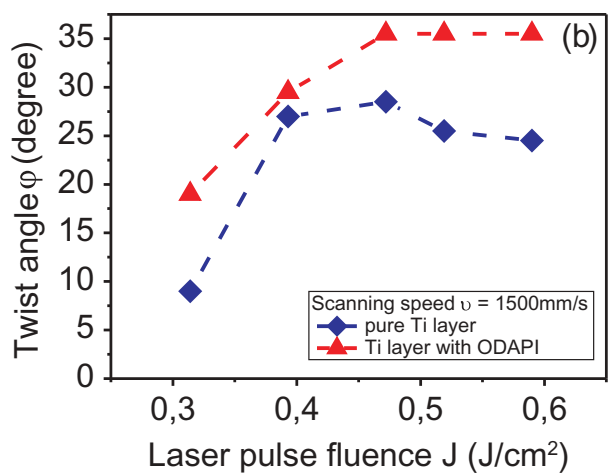

Figure 2. (Colour online) Dependence of the twist angle $\varphi$ of the LC cell on: (a) the scanning speed $v$ at a constant LPF $J=0.55 \mathrm{~J} / \mathrm{cm}^{2}$ and (b) the LPF $J$ at a constant scanning speed $v=1500 \mathrm{~mm} / \mathrm{s}$ during the processing of the Ti layer with the NLL method. The LC cell consists of the tested substrate having the pure NSTL (closed 'blue' squares and diamonds) and NSTL coated with the ODAPI film (closed 'red' circles and triangles). The dashed line is a guide to the eye. 
the processing of the Ti layer, by using measured twist angles, is shown in Figure 3 (closed 'blue' triangles). It is seen that for the pure NSTL the AE reaches the value $\sim 4.6 \times 10^{-6} \mathrm{~J} / \mathrm{m}^{2}$, which is lower in comparison with the value, obtained by Berreman's theory [6], but it agrees nicely with the $\mathrm{AE}$ of photoaligning layers as was mentioned above. It is evident that the difference between values of anchoring energies, experimentally obtained with the use of the twist LC cell (Equation (2)) and from Berreman's calculation, is observed due to the fact that the theory does not take into account the interaction between the LC molecules and aligning surface. The same conclusion was made for the photoaligning surfaces $[38,39]$.

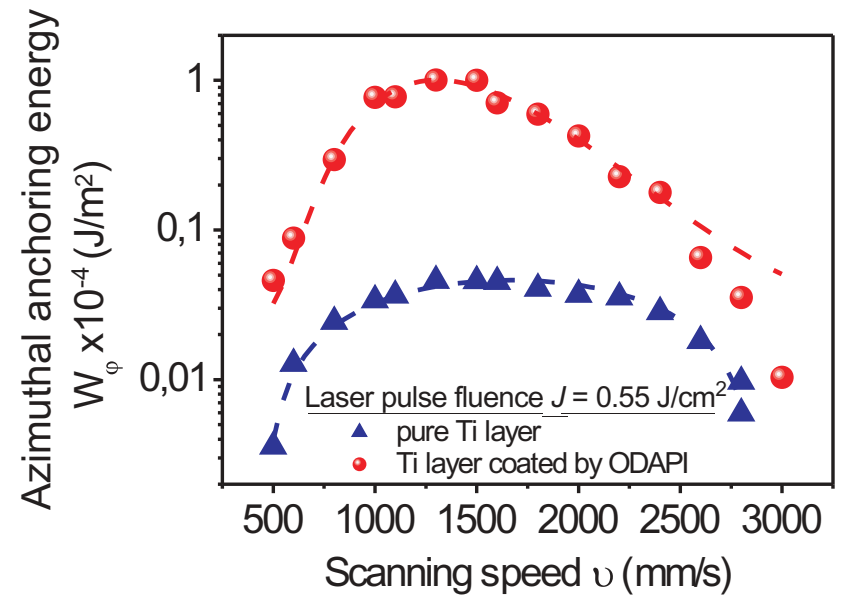

Figure 3. (Colour online) Dependence of the calculated azimuthal $A E$ (Equation (2)) of aligning films on the scanning speed $U$ during the processing of the Ti layer with the NLL method. The LC cell consists of the tested substrate having the pure NSTL (closed 'blue' triangles) and NSTL coated with the ODAPI film (closed 'red' spheres). The dashed line is a guide to the eye.
In the case of the NSTL with the coated ODAPI film the dependence of $\mathrm{AE}$ on the scanning speed $v$ is shown in Figure 3 with closed 'red' spheres. It is seen that coating of the polymer film onto the NSTL leads to the dramatic increase in the AE value $\sim 1 \times 10^{-4} \mathrm{~J} / \mathrm{m}^{2}$. It is obvious that this azimuthal $\mathrm{AE}$ consists of at least two components, namely: on the one hand, $\mathrm{AE}$, caused by the nanostructured layer with a certain period and depth and, on the other hand, owing to physical and chemical properties of the surface of a polymer and its interaction with LC molecules.

In addition, the influence of the LPF $J$ during the processing of the Ti layer at a certain constant scanning speed $v$ on $\mathrm{AE}$ values is shown in Figure 4. As can be seen from Figure 4, for each constant scanning speed $v$ there is an optimal value of the LPF $J$ when the maximal value of $\mathrm{AE}$ is reached. As can be seen from Figure 4(b), the gain of $\mathrm{AE}$ to the value $\sim 1 \times 10^{-4} \mathrm{~J} / \mathrm{m}^{2}$ can be reached after the processing of the Ti layer with the scanning speed $v=1500 \mathrm{~mm} / \mathrm{s}$ and LPF $J$ changing within a range $0.472-0.55 \mathrm{~J} / \mathrm{cm}^{2}$ and further deposition of the polymer ODAPI. It follows from Figure 4 that during the structuring of the Ti layer the choice of both the scanning speed and LPF allows changing the value of AE within a wide range $\sim 10^{-6}-10^{-4} \mathrm{~J} / \mathrm{m}^{2}$.

\section{Conclusions}

In conclusion to this letter, it should be noted that for the first time the NLL method as a new technique of the alignment of nematic LCs was proposed. We are demonstrating the possibility of the $\mathrm{AE}$ increasing of the pure $\mathrm{Ti}$ layers, owing to the coating of a polymer film onto the nanostructured surfaces. It was shown that the pure NSTL is characterised with the relatively weak $\mathrm{AE}$, while the $\mathrm{Ti}$ surface with the deposited polymer films has the strong AE. It was shown that the value of $\mathrm{AE}$ in a wide range can be controlled by means of the changing at least two
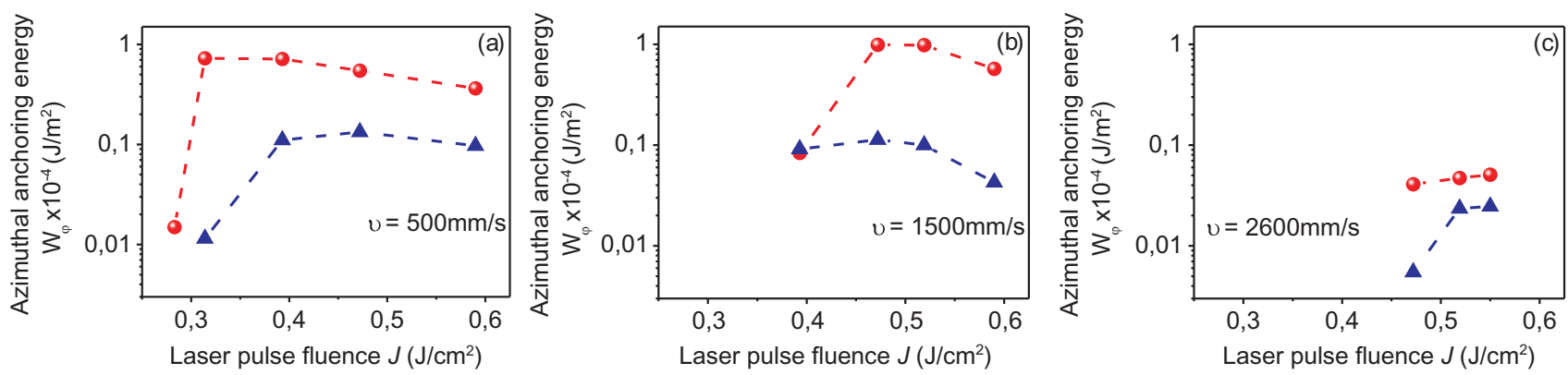

Figure 4. (Colour online) Dependence of the calculated azimuthal AE (Equation (2)) of aligning films on the LPF $J$ at constant scanning speed $v$ : (a) $500 \mathrm{~mm} / \mathrm{s}$, (b) $1500 \mathrm{~mm} / \mathrm{s}$ and (c) $2600 \mathrm{~mm} / \mathrm{s}$ during the processing of the Ti layer with the NLL method. The LC cell consists of the tested substrate having the pure NSTL (closed 'blue' triangles) and NSTL coated with the ODAPI film (closed 'red' spheres). The dashed line is a guide to the eye. 
parameters (scanning speed and LPF) during the structuring of the Ti layer and further coating of a polymer film without additional processing. In addition, on the basis of our studies described in this letter, we can propose a recipe to increase the azimuthal $\mathrm{AE}$ of the aligning $\mathrm{Ti}$ layer processed by means of NLL. For this purpose, there are two ways: (1) changing the parameters of NLL technique under processing of metal-layer, which will result in the decreasing of the period of NSTL and (2) the choice of a polymer, possessing stronger azimuthal AE. For instance, instead of ODAPI, which after some number of rubbing possesses $\mathrm{AE} \sim 1 \times 10^{-4} \mathrm{~J} / \mathrm{m}^{2}$ [30], we can use PI2555, which has $\mathrm{AE} \sim(4 \pm 1) \times 10^{-4} \mathrm{~J} / \mathrm{m}^{2}[31,32]$, or polymers, which apply for photoalignment of LCs, by changing AE within a wide range by means of light $[4,5,11,16]$.

\section{Acknowledgments}

The authors thank W. Becker (Merck, Darmstadt, Germany) for his generous gift of nematic liquid crystal E7, Prof. I. Gerus (Institute of Bio-organic Chemistry and Petrochemistry, NAS of Ukraine) for the kind provision of polymer ODAPI, Prof. O. Lavrentovich and his PhD student B. Li (Kent State University, USA) for gift of the polyimide PI2555 (HD MicroSystems, USA), Dr. S. Lukyanets (Institute of Physics, NAS of Ukraine) for the helpful discussions. We thank M. Gure and E. Karaman (Bilkent University, Turkey) for technical support of the NLL method. Ihor Pavlov and Fatih Ö. Ilday acknowledge financial support from European Research Council (ERC) Consolidator, Grant "Nonlinear Laser Lithography", No: ERC-617521 NLL. The authors also thank Mr. Reynaldo M. Bullon (the Production Editor of Taylor \& Francis Journals) for a great help and especial attention during final preparation for publication of the Letter.

\section{Disclosure statement}

No potential conflict of interest was reported by the authors.

\section{Funding}

Ihor Pavlov and Fatih Ö. Ilday acknowledge financial support from European Research Council (ERC) Consolidator, Grant "Nonlinear Laser Lithography”, No: ERC-617521 NLL.

\section{References}

[1] Congard J. Alignment of nematic liquid crystals and their mixtures. London (New York): Gordon and Breach Science Publishers; 1982.

[2] Ishimura K. Photoalignment of liquid-crystals systems. Chem Rev. 2000;100:1847-1874.

[3] O'Neill M, Kelly SM. Photoinduced surface alignment for liquid crystal displays. J Phys D: Appl Phys. 2000;33: R67-R84.
[4] Yaroshchuk O, Reznikov Y. Photoalignment of liquid crystals: basic and current trends. J Mater Chem. 2012;22:286-300.

[5] Seki T. New strategies and implications for the photoalignment of liquid crystalline polymers. Polymer Journal. 2014;46:751-768.

[6] Berreman DW. Solid surface shape and the alignment of an adjacent nematic liquid crystal. Phys Rev Lett. 1972;28:1683-1686.

[7] Berreman DW. Alignment of liquid crystals by grooved surfaces. Mol Cryst Liq Cryst. 1973;23:215231.

[8] Chatelain P. Orientation of liquid crystals by rubbed surfaces. C R Acad Sci. 1941;231:875-876.

[9] Sluckin TJ, Dunmur DA, Stegemeyer H. Crystal that flow. Classic papers from history of liquid crystals. London: Taylor \& Francis; 2004.

[10] Yasutake A, Oshiro RK, France patent application 7536984. 1975 Dec 3.

[11] Ishimura K, Suzuki Y, Seki T, et al. Reversible change in alignment mode of nematic liquid crystals regulated photochemically by command surfaces modified with an azobenzene monolayer. Langmuir. 1988;4:12141216.

[12] Gibbons WM, Shannon PJ, Sun ST, et al. Surfacemediated alignment of nematic liquid crystals with polarized laser light. Nature. 1991;351:49-50.

[13] Schadt M, Shmitt K, Kozinkov V, et al. Surface-induced parallel alignment of liquid crystals by linearly polymerized photopolymers. Jpn J Appl Phys. 1992;31:21552164.

[14] Dyadyusha A, Kozinkov V, Marusii T, et al. Lightinduced planar alignment of nematic liquid-crystal by the anisotropic surface without mechanical texture. Ukr Fiz Zh. 1991;36:1059-1062.

[15] Dyadyusha AG, Marusii T, Reshetnyak V, et al. Orientational effect due to a change in the anisotropy of the interaction between a liquid crystal and a bounding surface. JETF Lett. 1992;56:17-21.

[16] Chigrinov VG, Kozenkov VM, Kwok HS. Photoalignment of liquid crystalline materials: physics and applications. England: A John Wiley \& Sons, Ltd., Publication; 2008.

[17] Yaroshchuk O, Kravchuk R, Dobrovolskyy A, et al. Planar and tilted uniform alignment of liquid crystals by plasma-treated substrates. Liq Cryst. 2004;31:859869.

[18] Yaroshchuk O, Zakrevskyy Y, Dobrovolskyy A, et al. Liquid crystal alignment on the polymer substrates irradiated by plasma beam. In: Klimusheva GV, Iljin AG, editors. Nonlinear Optics of Liquid and Photorefractive Crystals 2000: Proceedings of Eighth International Conference on Nonlinear Optics of Liquid and Photorefractive Crystals. Proceedings of SPIE 4418; 2000 Oct 2-6; Alushta; Crimea (Ukraine); 2001. DOI:10.1117/12.428331

[19] Lee C-R, Fu T-L, Cheng K-T, et al. Surface-assisted photoalignment in due-doped liquid-crystal films. Phys Rev E. 2004;69:031704-1-6.

[20] Fuh AY-G, Liu C-K, Cheng K-T, et al. Variable liquid crystal pretilt angles generated by photoalignment in 
homeotropically aligned azo dye-doped liquid crystals. Appl Phys Lett. 2009;95:161104-1-3.

[21] Tennant DM, Koch TL, Mulgew PP, et al. Characterization of near-field holography grating masks for optoelectronics fabricated by electron beam lithography. J Vac Sci Technol B. 1992;10:2530-2535.

[22] Kim JH, Yoneya M, Yakoyama H. Tristable nematic liquid-crystal device using micropatterned surface alignment. Nature. 2002;420:159-162.

[23] Chou SY, Krauss PR, Renstrom PJ. Imprint lithography with 25-nanometer resolution. Science. 1996;272:85-87.

[24] Hill KO, Malo B, Bilodeau F, et al. Bragg gratings fabricated in monomode photosensitive optical fiber by UV exposure through a phase mask. Appl Phys Lett. 1993;62:1035-1037.

[25] Lin T-C, Huang L-C, Chou T-R, et al. Alignment control of liquid crystal molecules using crack induced selfassembled grooves. Soft Matt. 2009;5:3672-3676.

[26] Malinauskas M, Žukauskas A, Hasegawa S, et al. Ultrafast laser processing of materials: from science to industry. Light Sci Appl. 2016;5:e16133-13.

[27] Öktem B, Pavlov I, Ilday S, et al. Nonlinear laser lithography for indefinitely large-area nanostructuring with femtosecond pulse. Nat Photonics. 2013;7:897-901.

[28] Andrienko D, Kurioz Y, Nishikawa M, et al. Control of the anchoring energy of rubbed polyimide layers by irradiation with depolarized UV light. Jpn J Appl Phys. 2000;39:1217-1220.

[29] Gerus I, Glushchenko A, Kwon S-B, et al. Anchoring of a liquid crystal on photoaligning layer with varying surface morphology. Liq Cryst. 2001;28:1709-1713.

[30] Gvozdovskyy I. Electro- and photoswitching of undulation structures in planar cholesteric layers aligned by a polyimide film possessing various values of anchoring energy. Liq Cryst. 2017;xx:1-17. Published online. DOI:10.1080/02678292.2017.1359691.

[31] Senyuk BI, Smalyukh II, Lavrentovich OD. Undulations of lamellar liquid crystals in cells with finite surface anchoring near and well above the threshold. Phys Rev E. 2006;74:011712-1-3.

[32] Smalyukh II, Lavrentovich OD. Anchoring-mediated interaction of edge dislocations with bounding surfaces in confined cholesteric liquid crystals. Phys Rev Lett. 2003;90:085503-1-4.

[33] Rynes EP, Brown CV, Strömer JF. Method for the measurement of the $\mathrm{K}_{22}$ nematic elastic constant. App Phys Lett. 2003;82:13-15.

[34] Kozenkov Y, Chigrinov VG, Kwon SB. Photoanisotropic effects in poly(vinyl-cinnamate) derivatives and their applications. Mol Cryst Liq Cryst. 2004;409:251-267.

[35] Bryan-brown GP, Sage IC. Photoinduced ordering and alignment properties of polyvinylcinnamates. Liq Cryst. 1996;20:825-829.

[36] Voloshchenko D, Khizhnyak A, Reznikov Y, et al. Control of an easy-axis on nematic-polymer interface by light action to nematic bulk. Jpn J Appl Phys. 1995;34:566-571.

[37] Vorflusev VP, Kitzerow H-S, Chigrinov VG. Azimuthal anchoring energy in photoinduced anisotropic films. Jpn J Appl Phys. 1995;34:L1137-L1140.

[38] Kumar S, Kim JH, Shi Y. What aligns liquid crystals on solid substrates? The role of surface roughness anisotropy. Phys Rev Lett. 2005;94:077803-077804.

[39] Cull B, Shi Y, Kumar S, et al. Anisotropic surface morphology of poly(vinyl 4-methoxy-cinnamate) and 12-8 (poly)diacetylene thin films induced by linear photopolymerization. Phys Rev E: Stat Phys Plasmas Fluids Relat Interdiscip Top. 1996;53:3777-3781. 